\title{
Disruption of brain connectivity in acute stroke patients with early impairment in consciousness
}

\author{
Yuan-Hsiung Tsai ${ }^{1,2}$, Rui Yuan ${ }^{3}$, Yen-Chu Huang ${ }^{4}$, Mei-Yu Yeh $^{2}$, Ching-Po Lin ${ }^{1,5}$ *, Bharat B. Biswal ${ }^{3}$ * \\ ${ }^{\prime}$ Department of Biomedical Imaging and Radiological Sciences, National Yang-Ming University, Taipei, Taiwan \\ ${ }^{2}$ Department of Diagnostic Radiology, Chang Gung Memorial Hospital at Chiayi, College of Medicine and School of Medical Technology, Chang-Gung University, \\ Taoyuan, Taiwan \\ ${ }^{3}$ Department of Biomedical Engineering, New Jersey Institute of Technology, Newark, NJ, USA \\ ${ }^{4}$ Department of Neurology, Chang Gung Memorial Hospital at Chiayi, College of Medicine and School of Medical Technology, Chang-Gung University, Taoyuan, \\ Taiwan \\ ${ }^{5}$ Institute of Neuroscience, National Yang-Ming University, Taipei, Taiwan
}

\section{Edited by:}

Ursula Voss, Rheinische

Friedrich-Wilhelms University Bonn, Germany

\section{Reviewed by:}

Ursula Voss, Rheinische

Friedrich-Wilhelms University Bonn,

Germany

Daniel Collerton, Northumberland,

Tyne and Wear NHS Foundation

Trust, UK

\section{${ }^{*}$ Correspondence:}

Ching-Po Lin, Institute of

Neuroscience, National Yang-Ming

University, 155 Li-Nong Street,

Section 2, Peitou, Taipei, Taiwan

e-mail:ChingPoLin@gmail.com;

Bharat B. Biswal, Department of

Biomedical Engineering, New Jersey

Institute of Technology, 607 Fenster

Hall, University Heights, Newark, NJ

07102, USA

e-mail: bbiswal@gmail.com
Impairment in consciousness is common in acute stroke patients and is correlated with the clinical outcome after stroke. The underlying mechanism is not completely understood, with little known about brain activity and connectivity changes in acute stroke patients having impaired consciousness. In this study, we investigated changes in regional brain activity and brain networks of consciousness impaired stroke patients, as well as the amplitude of spontaneous low frequency fluctuation (ALFF) of each time series. Regional homogeneity (ReHo) of each voxel was measured, and resting state network analysis was consequently conducted. Results from this study demonstrate that, compared to normal subjects, the intensities of ALFF and ReHo, as well as the strength of the default mode network (DMN) connectivity, were significantly decreased in the precuneus and posterior cingulate cortex regions among stroke patients with impaired consciousness. Furthermore, the strength of the DMN was highly correlated with differences in the Glasgow Coma Scale (GCS) scores between the onset time and the scanning time. Results from this study suggest that the resting state $\mathrm{fMRI}$ is a feasible tool for the evaluation of acute stroke patients with an early impairment of consciousness. The detailed mechanisms, implications of these brain activities and networks exhibiting changes will require further investigation.

Keywords: fMRI, resting state, stroke, brain connectivity, resting state functional connectivity

\section{INTRODUCTION}

Early impairment of consciousness is common in acute stroke patients. A number of studies have demonstrated the existence of a relationship between the level of consciousness impairment and the mortality/morbidity rate after stroke (Frankel et al., 2000; Weir et al., 2003; Lansberg et al., 2011). An infarction or hemorrhage in anatomic regions that maintain arousal, such as the brainstem and the thalamus, may cause an early impairment of consciousness (Bogousslavsky et al., 1988; Amici, 2012). Large intracranial hemorrhaging or infarction with edema, and mass effect might also cause the midline shift. The degree of this shift has been shown to be correlated with the level of consciousness (Ropper, 1986). The proposed mechanism for such impairment has been associated with a direct destruction or compression of the reticular system (Steriade, 1996; Cucchiara et al., 2004). Although, some patients regain consciousness; others may have complications such as a prolonged coma or vegetative state. However, previous studies have yet to examine the altered state of consciousness in stroke patients and its relationship to the brain's activity and functional connectivity.

Recent advances in brain imaging techniques have expanded our understanding of how the brain functions. For example, changes in the blood oxygen level dependent (BOLD) signal during rest using functional magnetic resonance imaging (fMRI) have been shown to be highly associated with the connectivity of functionally related regions of the brain. Resting-state fMRI has been used to demonstrate spatiotemporal correlations within functional networks during rest (Biswal et al., 1995). One of the primary quantitative properties of the resting-state BOLD signal is the amplitude of low frequency fluctuations (ALFF), which measures the total power within the range of 0.01 and $0.1 \mathrm{~Hz}$ (Zang et al., 2007). Zang et al. (2007) found that children with ADHD showed reduced ALFF amplitude in specific and relevant regions of the brain. Similarly, Wang et al. (2012) found significant differences in ALFF in brain regions of patients with severe depression. We have previously shown that ALFF correlated significantly with hypercapnic fMRI response in healthy subjects (Kannurpatti et al., 2010; Di et al., 2012). These studies demonstrate the feasibility of using ALFF as a neurophysiological index to show group differences between healthy controls and a number of different clinical populations.

Another quantitative property of resting fMRI is regional homogeneity (ReHo), which characterizes the similarity of local brain activity across a region. In fMRI studies, this measure is obtained by measuring Kendall's coefficient of concordance (KCC) of a cubic cluster of 27 voxels, with the central voxel of every cubic cluster assigned the derived KCC value. ReHo has also been used 
in several clinical studies, including attention deficit hyperactivity disorder (ADHD; Wang et al., 2013), Alzheimer's disease (Zhang et al., 2012), and Parkinson's disease (Wu et al., 2009).

The default-mode network (DMN; Greicius etal., 2003) is a well-established functional network that is observed during the resting-state imaging. It is generally suppressed during task activation states such as those requiring attention (Uddin et al., 2010) and decision making (Buckner et al., 2008). The DMN is typically comprised of the posterior cingulate cortex (PCC), the precuneus, the inferior parietal, and the medial prefrontal cortex regions. Functional disconnection of the DMN tends to be associated quite strongly with an impairment of consciousness, since the DMN is important in the genesis of awareness (Vanhaudenhuyse et al., 2010; Soddu et al., 2011; Boly et al., 2012; Fernandez-Espejo et al., 2012).

The current study uses resting-state fMRI to achieve two specific goals. The first goal is to characterize the resting state signals by using specific quantitative measures, including ALFF and ReHo, in order to differentiate between stroke patients and health subjects. The second goal is to determine whether the properties of the DMN vary between stroke patients with impaired consciousness and normal healthy subjects. To assess these network properties, spatial independent component analysis (ICA) and dual regression are used to calculate the resting state functional connectivity (RSFC) parameters. We hypothesize that the early alteration of regional brain activity and DMN connectivity play a key role in determining conscious status of acute stroke patients, and that the resting fMRI approach may be used as an imaging marker to estimate the subject's conscious status and to predict the recovery of consciousness.

\section{MATERIALS AND METHODS SUBJECTS}

This study was part of an integrated stroke project at Chang Gung Memorial Hospital, Taiwan, and was approved by the Institutional Review Board of Chang Gung Memorial Hospital. Informed consents were obtained from all the patients' relatives. Fifteen unconscious patients suffering from their first stroke with no history of any neurological deficits were enrolled. Patients with any contraindications to undergoing the MRI scans and patients requiring emergency surgery were excluded. The Glasgow Coma Scale (GCS) scores and motor response scores were estimated by an experienced emergency physician within $24 \mathrm{~h}$ of the stroke. The demographic and clinical characteristics for the included stroke subjects are presented in Table 1. In addition, 19 healthy subjects (age range from 77 to 88 years, average $81+4.11$ ) without a history of neurological disease or any pathological findings in conventional MRI scans were enrolled.

\section{MRI INSTRUMENTATION AND PROCEDURES}

All data were collected using a $3 \mathrm{~T}$ Siemens Vario MRI system (Siemens Medical System, Erlangen, Germany) with a 32 channel head coil. The MRI scans were all performed within $24 \mathrm{~h}$ of the stroke. The imaging procedure for each subject included obtaining T1-weighted anatomical images with a gradient echo sequence $(\mathrm{TR}=3500 \mathrm{~ms}, \mathrm{TE}=2.87 \mathrm{~ms}$, FOV $=220 \mathrm{~mm}$, matrix $=256 \times 256 \times 160)$, resulting in a spatial resolution of $0.9 \mathrm{~mm} \times 0.9 \mathrm{~mm} \times 1.0 \mathrm{~mm}$. Additionally, a gradient EPI sequence, sensitive to BOLD contrast, was also obtained $(\mathrm{TR}=2500 \mathrm{~ms}, \mathrm{TE}=27 \mathrm{~ms}, \mathrm{FOV}=220 \mathrm{~mm}$, matrix $=64 \times 64 \times 36$, slice thickness $=4 \mathrm{~mm}$ ). Each scan consisted of 240 image volumes and lasted for $10 \mathrm{~min}$ and $7 \mathrm{~s}$. Healthy subjects were instructed to stay awake and relaxed with their eyes closed during the resting state scan.

\section{IMAGE PROCESSING}

All fMRI data from each of the subjects was processed using Analysis of Functional NeuroImages (AFNI) software ${ }^{1}$ and the SPM8 package $^{2}$. All data sets were processed using an image registration algorithm for the detection and correction of motion related signal changes. Any subject that exhibited head motion greater than $2 \mathrm{~mm}$ was discarded from further analysis. Furthermore, six motion parameters and their Euclidean norm of motion derivatives were regressed out from all time series of each subject. All time series were also linearly detrended to correct for any linear drifts. The anatomical images were partitioned into gray matter, white matter (WM) and cerebrospinal fluid (CSF). Each subject's deformation field map obtained from the structural image was applied to the functional images for normalization into the Montreal Neurological Institute (MNI) space (resampled at $3 \mathrm{~mm}^{3}$ resolution). For each time series, the first five principle components from both WM signals, and CSF signals were regressed out. A band-pass filter ranging from 0.01 to $0.1 \mathrm{~Hz}$ was applied to each time series. All data sets were then smoothed using an $8 \mathrm{~mm}$ FWHM filter.

\section{ALFF MAP}

Amplitude of spontaneous low frequency fluctuation was calculated using the Resting-State fMRI Data Analysis Toolkit V1.7 $(\text { REST) })^{3}$. For each voxel, a discrete Fourier transform was performed on the resting-state time series. The ALFF was computed by measuring the average square root of the total power spectrum between 0.01 and $0.10 \mathrm{~Hz}$ on a voxel by voxel basis.

\section{ReHo MAP}

Regional homogeneity was calculated in a voxel-wise manner using KCC among the time series of a centered cubic cluster of 27 voxels. A large ReHo value for a given voxel indicates a high local synchronization of resting state fMRI signal among neighboring voxels, and vice versa.

\section{INDEPENDENT COMPONENT ANALYSIS}

Independent component analysis was used to study the resting state network patterns across brain. First, the resting scans of all healthy controls were concatenated along the time axis into a single $4 \mathrm{D}$ dataset. This $4 \mathrm{D}$ dataset was decomposed by MELODIC into 20 spatio-temporal components, a set of statistically independent sources of the resting state signal represented by large-scale patterns of co-activated voxels. Based on visual comparison with previously identified resting state network patterns (Biswal et al., 2010; Cole etal., 2010), 10 RSN components were selected for further analysis.

\footnotetext{
${ }^{1}$ http://afni.nimh.nih.gov/afni; version: January 2012

${ }^{2}$ http://www.fil.ion.ucl.ac.uk/spm/ (date last accessed 13 January 2012)

${ }^{3}$ http://www.restfmri.net (date last accessed 25 May 2012)
} 


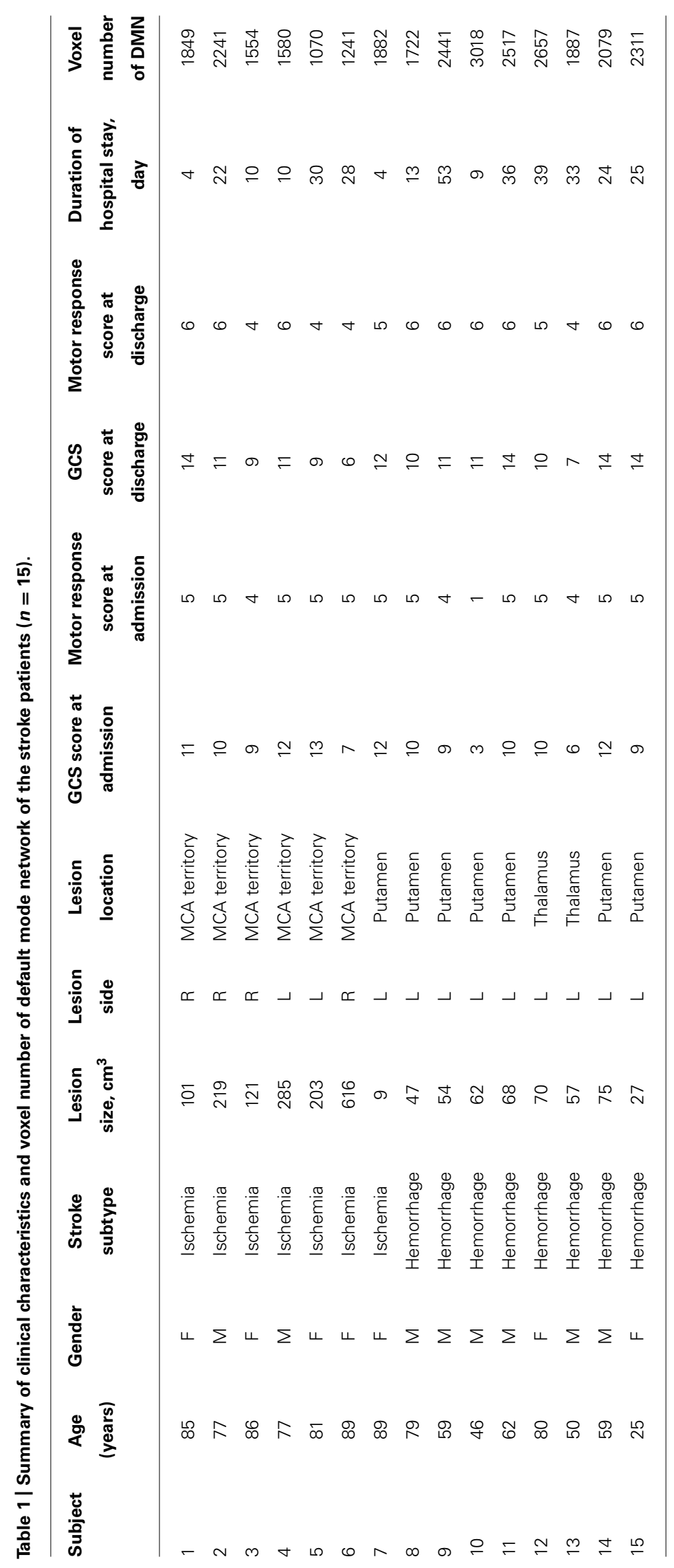


Dual regression was used to derive individual restingstate networks for each subject from each group component (Beckmann et al., 2005). Each spatially independent component map produced in the group-level ICA has a corresponding temporal signal. These temporal signals are used in a second regression for each individual in order to identify the spatial map per subject identifiable with that group component. Following standard steps, the regression betas from the individual level RSNs were then transformed to $Z$-scores.

\section{STATISTICAL ANALYSIS}

To determine group differences ALFF, ReHo and functional network connectivity, a two-sample $t$-test was conducted within a general linear model framework. This was used to determine locations of voxels of significant differences between stroke patients and healthy subjects within the DMN, such as within the precuneus and the temporo-parietal junction. Analyses of correlation between clinical measurement and DMN voxel number were performed with bivariate correlations. The correlations were presented with Spearman rank correlation coefficients. A p-value of less than 0.05 was deemed as a significant correlation. All statistical analyses were performed using Statistical Product and Service Solutions (SPSS, Version 18).

\section{RESULTS}

\section{AMPLITUDE OF LOW FREQUENCY FLUCTUATIONS AND REGIONAL HOMOGENEITY ANALYSIS}

Figure 1 demonstrates differences in ALFF (Figure 1A) and ReHo (Figure 1B) between healthy subjects and stroke patients with impaired consciousness. Significant group differences appear in the precuneus for ReHo values in the PCC for ALFF. Other brain regions do not reveal significant difference between these two groups. Both the precuneus and PCC regions are considered to be inside the DMN, and highly involved with states of consciousness.

The slight difference in results between ALFF and ReHo may be attributable to previous findings that ALFF maps are more vulnerable to the effects of large vessels than ReHo maps (Di et al., 2012; Yuan et al., 2013). Moreover, several voxels in the medial frontal cortex (MFC) region also show significant differences in ALFF but not in ReHo. The reason may be ALFF measures the power of time series from voxel to voxel, while ReHo measures similarity, which puts all neighboring voxels into consideration. Thus ALFF detects voxel-wise signal differences in the ALFF; ReHo differences represent differences in connectivity among a small group of neighboring voxels.

\section{RESTING STATE NETWORK ANALYSIS}

In Figures 2A,B show the spatial extent of the DMN for the healthy subjects and stroke patients with impaired consciousness. Figure 2C illustrates significant differences in the spatial extent of the DMN between the two groups. Among patients with impaired consciousness, a significant reduction in the activity of the PCC, the precuneus and the inferior parietal cortex can be observed (Figures 2D,E).

Considering that the results of changes in DMN connectivity in this study may be mainly due to the effect of stroke instead of conscious impairment, we also investigated the differences of other resting state networks (Figure 3). The networks that show significant differences, including the executive network and the attention network, are all related to consciousness and awareness. To further address the role of DMN in consciousness, we calculated the normalized volume of DMN for each subject.

\section{CORRELATION OF RSFC TO CLINICAL MEASUREMENTS}

The average volume of the DMN (measured by counting interior voxels in MNI space) was significantly lower in stroke patients with impaired consciousness than in healthy subjects $(p<0.001$; shown in Figure 4). There were no significant correlations between DMN voxel number and GCS at the time of the MRI scan $(R=-0.296$; $P=0.284)$ nor at the time when the patient was discharged $(R=0.468 ; P=0.078)$. A significant correlation between voxel number in the DMN with the difference of GCS at the time of the MRI scan and the time of the discharge $(R=0.720 ; P=0.002)$ was observed (shown in Figure 5).

\section{DISCUSSION}

Using resting-state $\mathrm{AMRI}$, this study compared the regional brain activity between stroke patients with impaired consciousness and normal healthy subjects by measuring LFFs and the DMN connectivity. To the best of our knowledge, this is the first study using ALFF and RSFC to evaluate brain activity and DMN connectivity of stroke patients with impaired consciousness. Stroke patients in this study had suffered localized brain damage via ischemia or hematoma. Damage from these phenomena is significantly different than that caused by traumatic brain injury or hypoxic brain injury, which are commonly reported in prior studies of conscious disorder. Additionally, vascular pathologies, such as hypertensive angiopathy, amyloid artheriopathy atherosclerosis, or arthritis, are often encountered in stroke patients (Gregoire et al., 2011). These vascular lesions may affect the BOLD signals, thus fMRI studies of stroke patients with impaired consciousness may improve our understanding of consciousness as a whole.

Early impairment of consciousness is associated with poor functional outcome for stroke patients, and acute impairment of consciousness affects whether patients recover or enter a prolonged coma or a vegetative statue. Thus, further understanding of how brain activity and connectivity change after stroke onset and how it affects the patient's consciousness is important.

\section{ALTERED AMPLITUDE OF LOW FREOUENCY FLUCTUATIONS IN STROKE PATIENTS WITH IMPAIRMENT OF CONSCIOUSNESS}

In contrast to the functional connectivity methods that measure temporal synchronization between brain regions, the ALFF and ReHo approaches performed here localize the brain's functional alterations. These analyses provide additional information to understand the disturbances of neural networks demonstrated in fMRI studies in patients with impaired consciousness. Using both the ALFF and ReHo measures, we found that among the stroke patients with impaired consciousness, the ALFFs were significantly reduced mainly in the precuneus and adjacent PCC regions. 


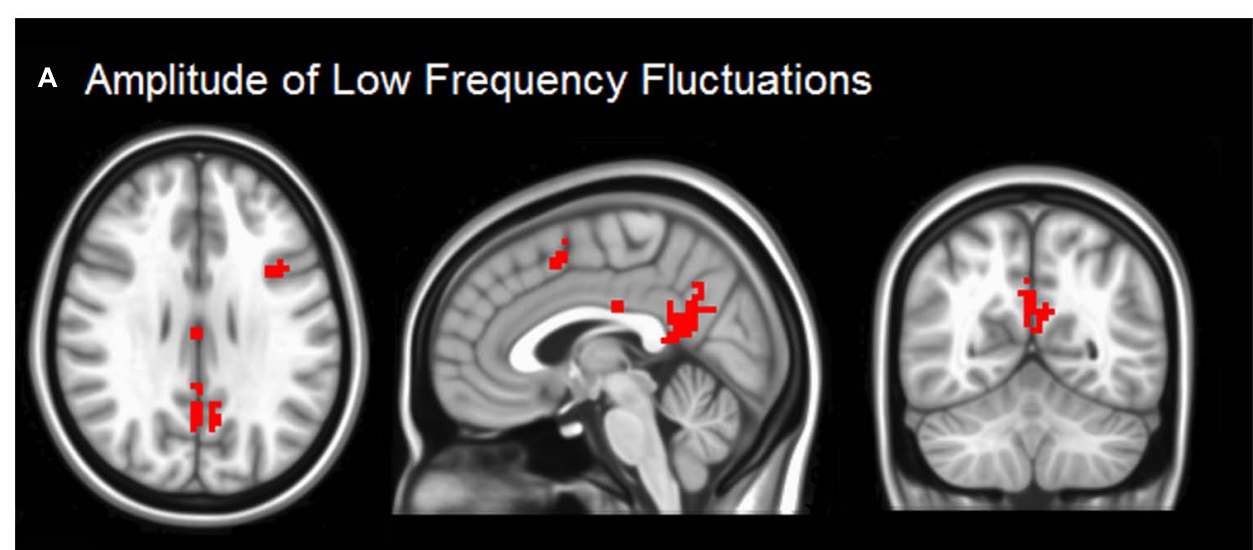

\section{B Regional Homogeneity}
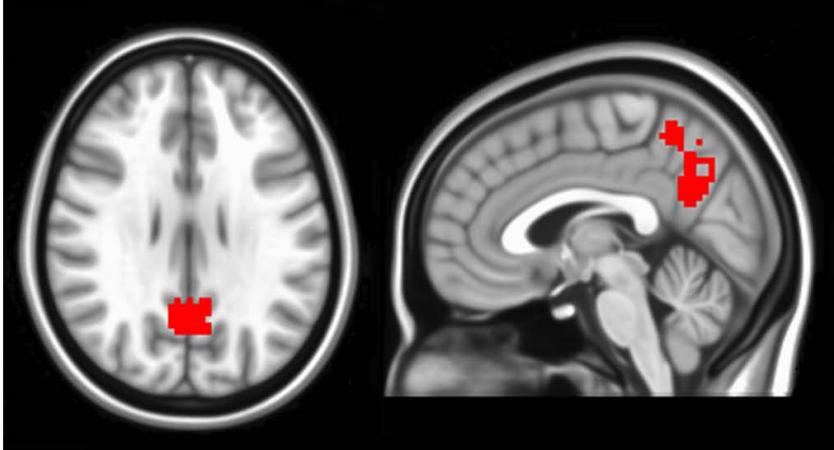

\section{c Overlap between ALFF and ReHo}
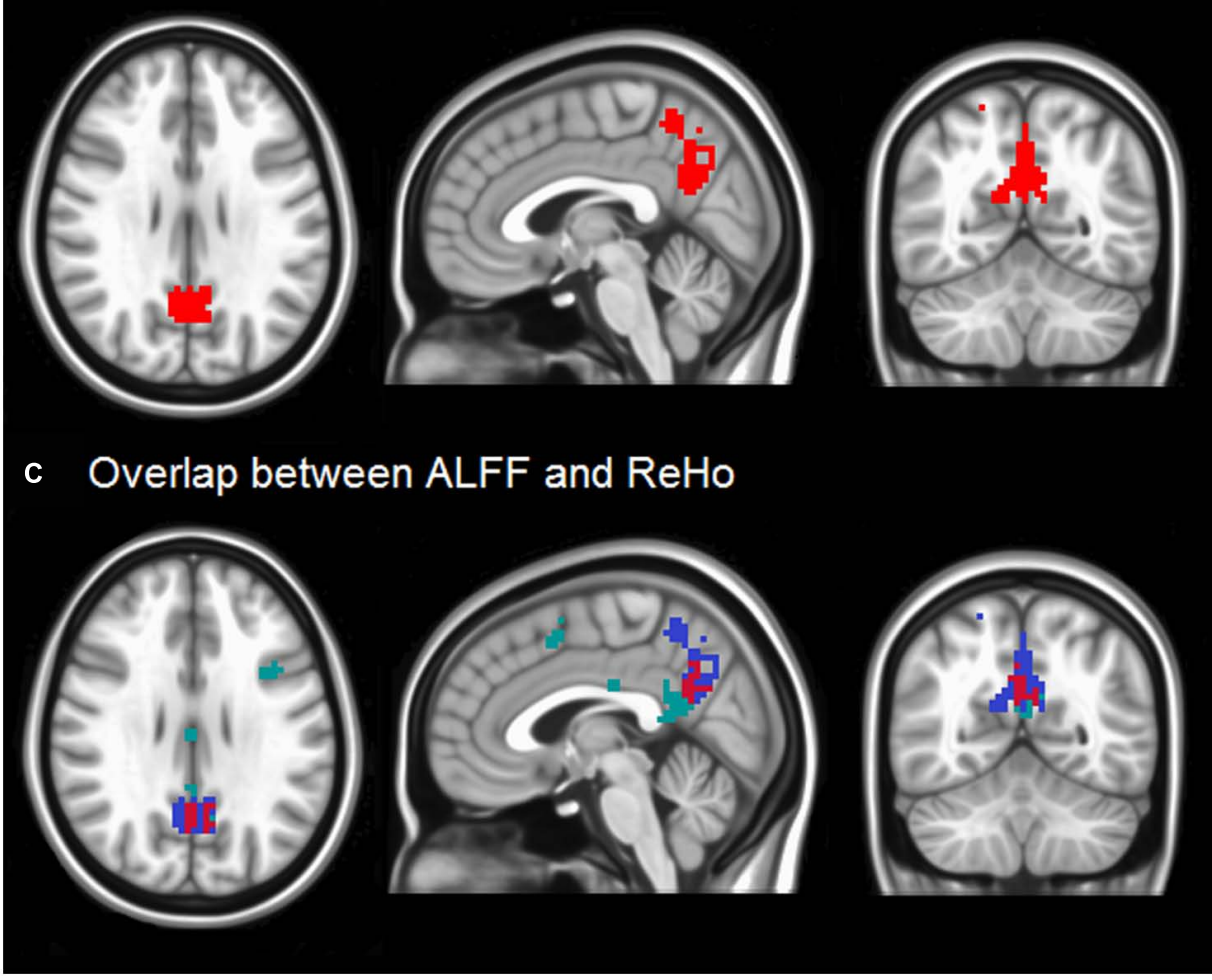

FIGURE 1 | (A) The two sample $t$-test result of ALFF between two groups with the threshold at corrected $p<0.05$ is shown. The regions around PCC (highlighted in red) indicate that stroke patients with impaired consciousness have lower ALFF than matched normal controls. (B) Two sample $t$-test results of ReHo between two groups at $p<0.05$ is shown. The results were threshold at corrected $p<0.05$. The red regions imply that stroke patients with impaired consciousness have lower regional homogeneity than the normal controls. (C) The overlaps between ALFF results and ReHo results are in the red region. The green and blue regions show unique ALFF and unique ReHo, respectively.
The precuneus is among the most active cortical regions in the DMN during conscious resting state, and along with the adjacent PCC, has been known to show deactivation in a number of pathophysiological measures of altered states of consciousness. For instance, the precuneus, along with the lateral parietal and prefrontal cortices, was found to be significantly less active during slow-wave and rapid eye movement sleep than during rest (Maquet et al., 1996, 1999). Since the impaired consciousness of self and the environment represents a key feature shared by the different sleep stages, these observations might provide evidence for the role of the precuneus in conscious processes. Anesthetic agents have been found to decrease cerebral blood flow to the precuneus in a dose dependent manner (Kaisti et al., 2002). Another study showed disruption within the functional networks of the PCC-precuneus region and within the non-specific thalamus during general anesthesia (Hudetz, 2012). These studies indicate that anesthetics result in behavioral changes via an effect within specific neuronal networks that regulate arousal and consciousness, 


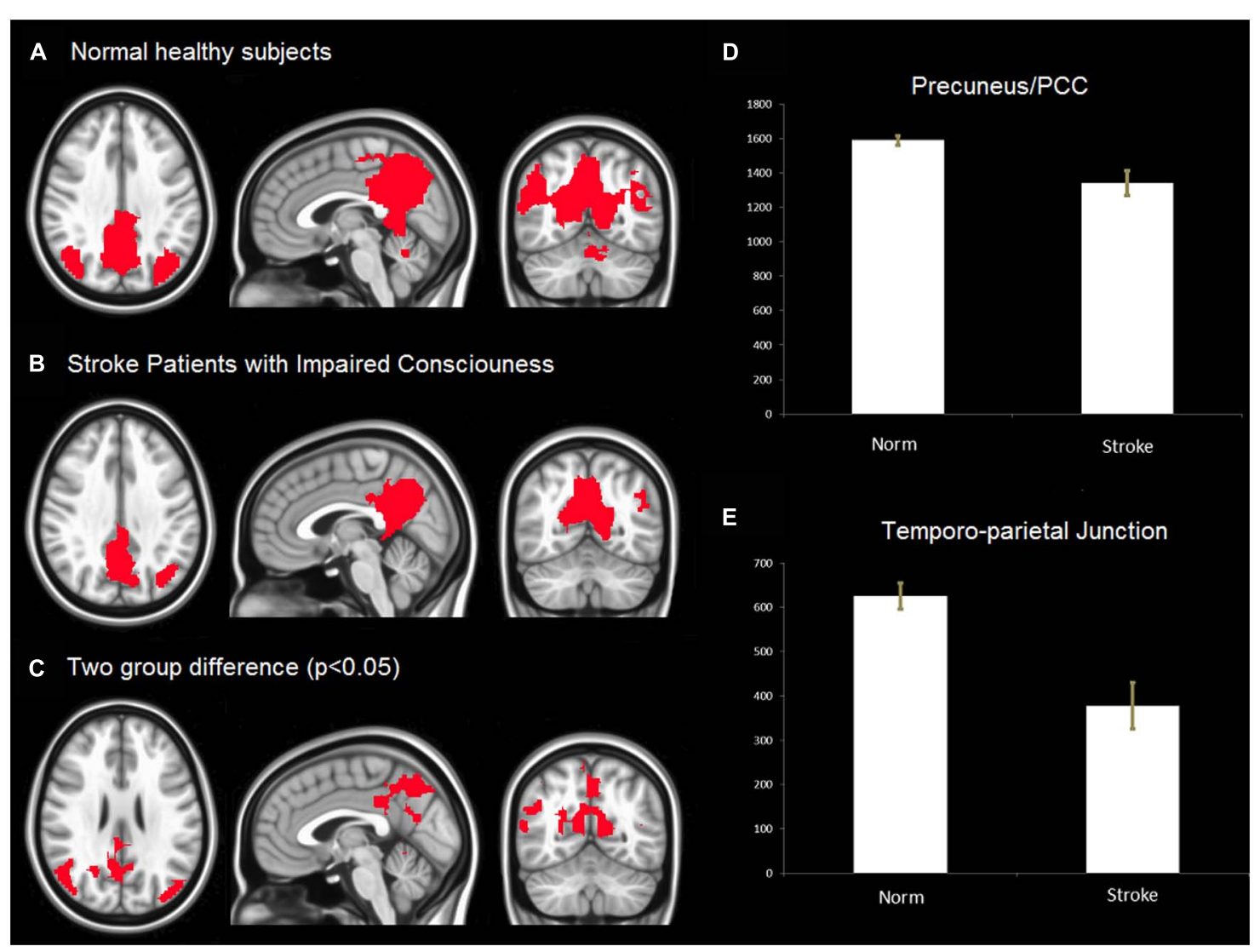

FIGURE 2 | (A) Default Mode Network (DMN) in healthy controls. The result was threshold at corrected $p<0.05$ rendered on MNI152 template. (B) DMN of stroke patients with impaired consciousness threshold at corrected $p<0.05$. The result was overlaid on MNI152 template. (C) The group difference of DMN. The result was threshold at corrected $p<0.05$ and rendered on MNI152 template. (D,E) voxels $(T>3.2)$ number of default mode network in PCC/Precuneus, temporo-parietal junction between stroke patient with impaired consciousness and healthy controls. The results show that the number of voxels $(T>3.2)$ of normal health is significantly more than that of stroke patients with impaired consciousness $(p<0.05$ ) in both $\mathrm{PCC} /$ precuneus and temporo-parietial junction. including the precuneus. Furthermore, in a study of hypnosis, it was found that relative cerebral blood flow decreased in the precuneus, PCC and right inferior parietal regions (Rainville et al., 1999). In particular, deactivation of the precuneus, was considered to be an important metabolic feature of this altered state of consciousness.

Taken together with the widespread connectivity pattern differences, these findings provide strong, albeit preliminary evidence that the networks to which the precuneus belong may be parts of the neural network serving self-awareness and conscious experience. The precuneus may play a central role in the functional network correlated to consciousness. In this study of stroke patients with impaired consciousness, we have reinforced the known importance of the activity of the precuneus and PCC in the genesis of awareness and maintenance of consciousness.

\section{DEFAULT MODE NETWORK DISRUPTION IN STROKE SUBJECTS WITH IMPAIRMENT OF CONSCIOUSNESS}

Using data-driven ICA, we find disrupted DMN connectivity among stroke patients with impaired consciousness. The connectivity among the PCC, the precuneus, the medial prefrontal, and the inferior parietal regions is decreased among stroke patients. High DMN connectivity has been consistently found in healthy subjects using ICA analysis of resting fMRI data (Meindl et al., 2010). Disruption of the DMN has been linked to pathophysiological conditions with various levels of reduced cognitive function, for instance in cases of epilepsy (Danielson et al., 2011), Alzheimer's disease (Greicius et al., 2004), depression (Greicius et al., 2007), and schizophrenia (Zhou et al., 2007). Disruption of the DMN was found to be associated with behavior measurement and cognitive performance. Studies of patients with severe traumatic brain injury, including minimally conscious and vegetative state patients, showed disruption of DMN connectivity (Boly et al., 2008; Vanhaudenhuyse et al., 2010; Sharp et al., 2011). The decreased connectivity between the medial prefrontal and PCC/precuneus areas has been observed in vegetative patients, with restored metabolism in the precuneus after conscious recovery (Laureys et al., 1999). Another recent study of patients with different levels of disordered consciousness showed significant impairment in all of the pathways within the DMN (Fernandez-Espejo et al., 2012). These previous studies, combined with the present results, support the conclusion that disruption of 


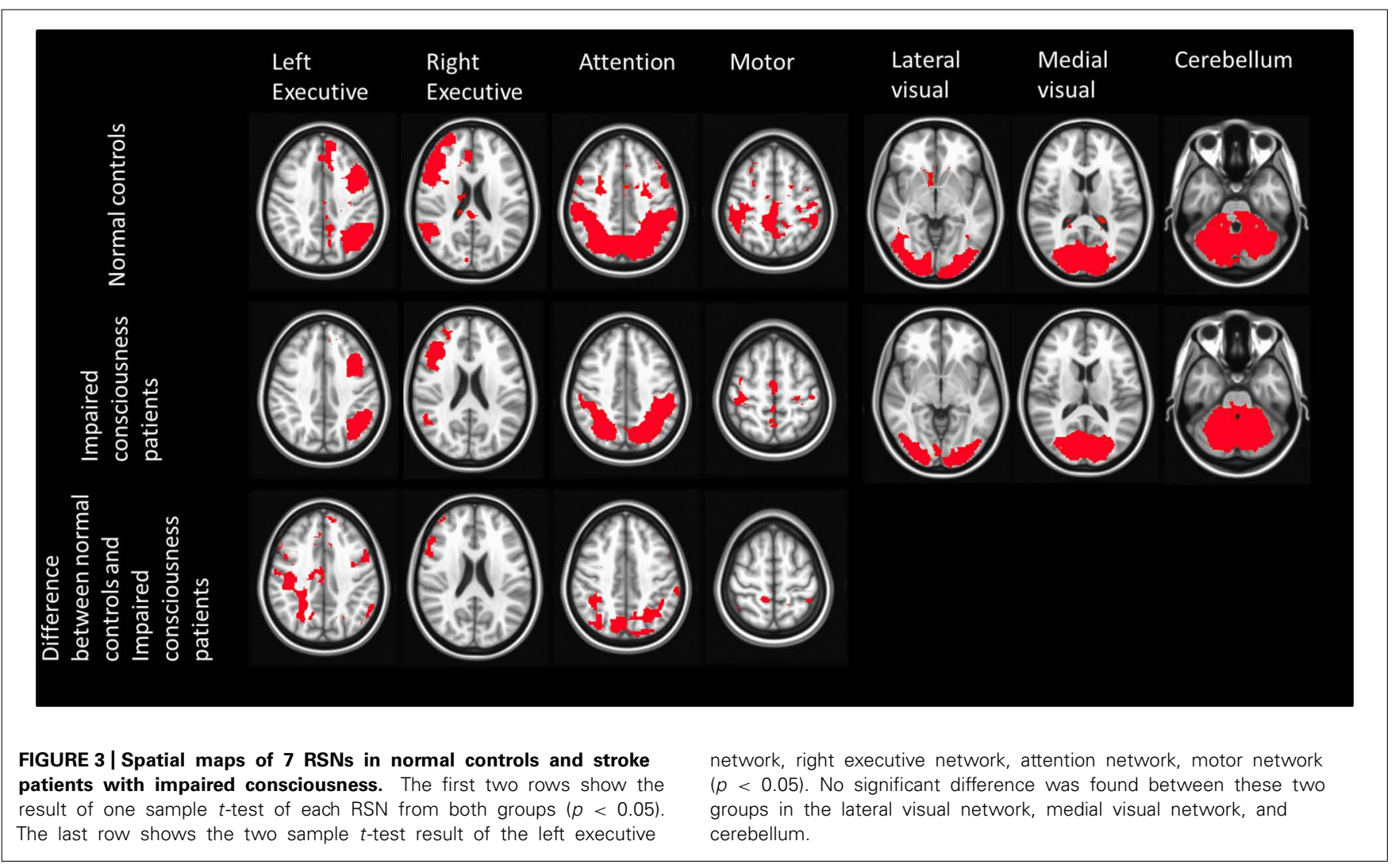

the DMN in stroke patients is associated with the impairment of consciousness, rather than with the alteration of cerebrovascular signal.

\section{DEFAULT MODE NETWORK CONNECTIVITY AND THE SEVERITY OF CONSCIOUS IMPAIRMENT}

The GCS is a reproducible and objective neurological scale that has been used to record the conscious state of a person for initial

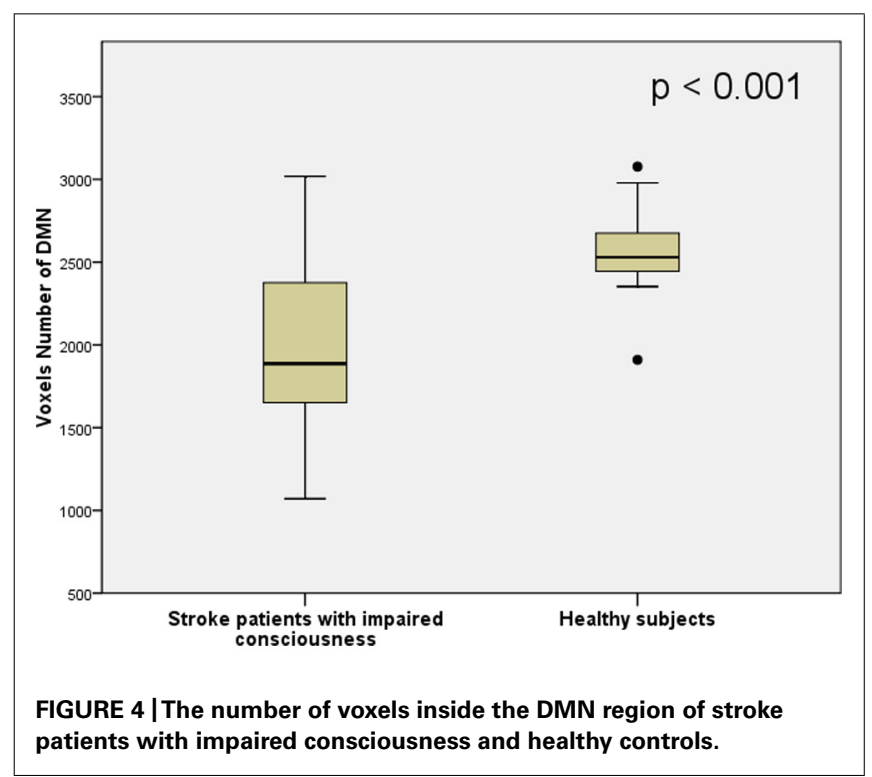

and subsequent assessment (Teasdale and Jennett, 1974). Initially used to assess level of consciousness after head injury, the GCS has become the method of choice after acute neurological insult, for documenting neurologic findings over time and for predicting functional outcome (Fischer and Mathieson, 2001). In the present study, the DMN volume was positively correlated with the change

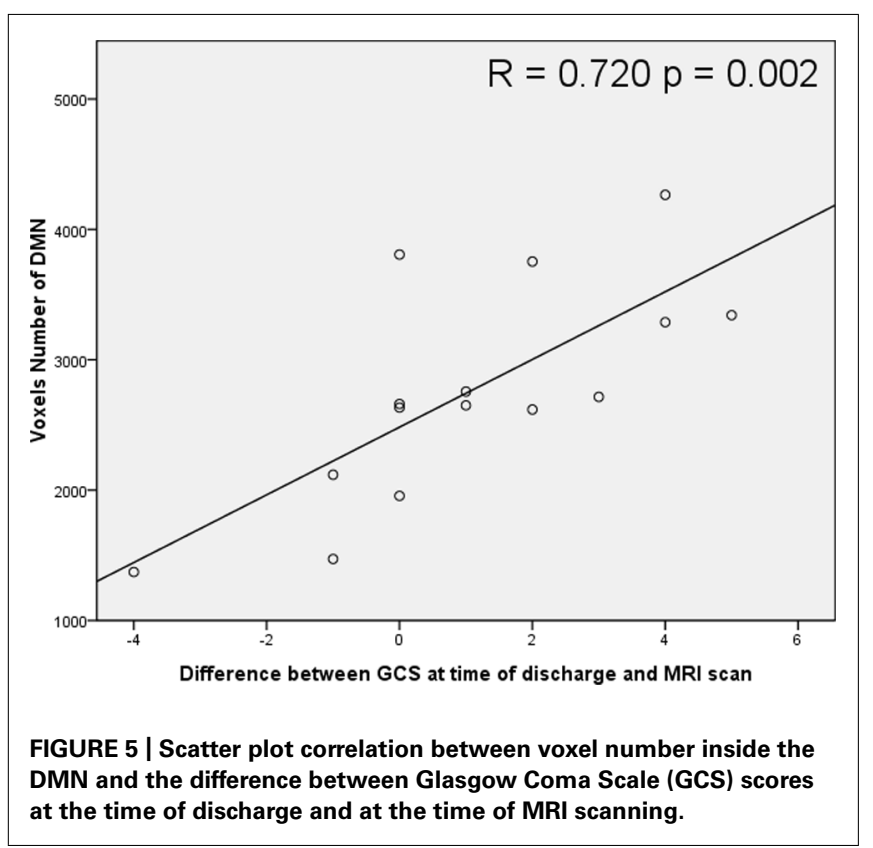


of GCS score over time (value at the time of discharge minus that at the time of the MRI scan).

Default mode network connectivity is correlated with the behavioral signs of awareness in patients with prolong conscious disorders such as minimally conscious state and vegetative state (Fernandez-Espejo et al., 2012; Soddu et al., 2012). To our knowledge, the present is the first study to show the correlation of GCS changes and DMN connectivity in acute neurological illness. It is important to note that the meaning of an impairment of consciousness is different in an acute stage than in a chronic stage (Ohta, 2005). Patients suffering from acute neurological illness such as an infarction, spontaneous hemorrhaging, or traumatic brain injury may experience transient or prolonged impairment of consciousness. The duration and the level of consciousness impairment during the acute stage are important factors for the patient's prognosis (Weir et al., 2003; McNett, 2007). However, in some patients, the level of consciousness is difficult to estimate. For example, patients who suffer from high cervical cord injury, or other severe limb injury cannot cooperate with the motor response measurement; aphasia or intubation prevents verbal responses from being accurately estimated, and severe eye injury does not allow eye opening/closing methods of evaluation (Barlow, 2012). From the results of this study, the DMN may be considered a promising marker for the prognosis of conscious status.

\section{DIFFERENCE OF NETWORK CONNECTIVITY BETWEEN STROKE AND HEALTHY SUBJECTS}

In a recent study, Park et al. (2011) studied the longitudinal effects of RSFC in stroke patients. To test the hypothesis that the alteration of DMN connectivity in stroke patients found in the present study may not be related to impairment of consciousness but instead to the effects of stroke, we also reanalyzed the other resting state networks with ICA method (Figure 3). Figure 3 demonstrates significant differences of network strength between stroke unconscious patients and healthy subjects. These changes were noted in bilateral executive, attention, and sensorimotor networks. On the contrary, no significant difference was found in medial and lateral visual and cerebellar networks. Thus, the DMN differences between stroke unconscious patients and healthy subjects appear to not be due solely to global vascular effects originating from stroke, as there were several networks in stroke patients had properties similar to those of the healthy subjects.

Moreover, the networks that showed differences in this study, including executive, attention, and DM networks, have also been reported to be related to consciousness and awareness in previous publications (Vanhaudenhuyse et al., 2010, 2011). In addition, the significant correlation of the networks strength with change of GCS between time of discharge and time of MRI scan was only obtained in DMN.

\section{LIMITATIONS AND FUTURE DIRECTIONS}

This study, since it is preliminary in nature, has several limitations. First, as we mentioned in the introduction section, stroke lesions in specific anatomic regions and large lesions with mass effect and midline shift are highly correlated with the level of consciousness. Thus, further investigations enrolling subjects with more specific lesion locations can clarify how the lesion locations disrupt brain network connections and LFFs. Second, a longitudinal study including resting state fMRI scans in a follow-up stage or after patients recover consciousness may be helpful in understanding brain plasticity following impairment of consciousness. In addition, it is necessary to further study whether the change of DMN is due mainly to the impairment of consciousness or to other physiological effects.

\section{CONCLUSION}

This resting state fMRI study's findings identify specific declines in ALFF and ReHo as well as disruptions of DMN in stroke patients with an early impairment of consciousness. In the context of previously reported decline of the regional blood flow and the disconnections of DMN, resting state fMRI has been shown to be a feasible tool for evaluating stroke patients with a conscious disorder. Further study of larger samples of patients in order to investigate the detailed mechanisms and predictive value of the changes in network connectivity to patient's outcome is necessary.

\section{ACKNOWLEDGMENTS}

We greatly appreciate the assistance of Ms. Dharni Patel and Dr. Paul Taylor for careful reading of the manuscript. This research was partly supported by the US National Institutes of Health grant 5R01AG032088 and the Chang Gung Medical Research Fund, Chang Gung Memorial Hospital, Taiwan CORPG690463 and CORPG690453.

\section{REFERENCES}

Amici, S. (2012). Thalamic infarcts and hemorrhages. Front. Neurol. Neurosci. 30:132-136. doi: 10.1159/000333611

Barlow, P. (2012). A practical review of the Glasgow Coma Scale and score. Surgeon 10, 114-119. doi: 10.1016/j.surge.2011.12.003

Beckmann, C. F., DeLuca, M., Devlin, J. T., and Smith, S. M. (2005). Investigations into resting-state connectivity using independent component analysis. Philos. Trans. R. Soc. Lond. B Biol. Sci. 360, 1001-1013. doi: 10.1098/rstb. 2005.1634

Biswal, B. B., Mennes, M., Zuo, X. N., Gohel, S., Kelly, C., Smith, S. M., etal. (2010). Toward discovery science of human brain function. Proc. Natl. Acad. Sci. U.S.A. 107, 4734-4739. doi: 10.1073/pnas.09118 55107

Biswal, B., Yetkin, F. Z., Haughton, V. M., and Hyde, J. S. (1995). Functional connectivity in the motor cortex of resting human brain using echo-planar MRI. Magn. Reson. Med. 34, 537-541. doi: 10.1002/mrm.1910340409

Bogousslavsky, J., Regli, F., and Uske, A. (1988). Thalamic infarcts: clinical syndromes, etiology, and prognosis. Neurology 38, 837-848. doi: 10.1212/WNL.38.6.837

Boly, M., Massimini, M., Garrido, M. I., Gosseries, O., Noirhomme, Q., Laureys, S., et al. (2012). Brain connectivity in disorders of consciousness. Brain Connect. 2, 1-10. doi: 10.1089/brain.2011.0049

Boly, M., Phillips, C., Tshibanda, L., Vanhaudenhuyse, A., Schabus, M., Dang-Vu, T. T., et al. (2008). Intrinsic brain activity in altered states of consciousness: how conscious is the default mode of brain function? Ann. N. Y. Acad. Sci. 1129, 119-129. doi: 10.1196/annals.1417.015

Buckner, R. L., Andrews-Hanna, J. R., and Schacter, D. L. (2008). The brain's default network: anatomy, function, and relevance to disease. Ann. N. Y. Acad. Sci. 1124, 1-38. doi: 10.1196/annals.1440.011

Cole, D. M., Smith, S. M., and Beckmann, C. F. (2010). Advances and pitfalls in the analysis and interpretation of resting-state FMRI data. Front. Syst. Neurosci. 4:8. doi: $10.3389 /$ fnsys. 2010.00008 
Cucchiara, B. L., Kasner, S. E., Wolk, D. A., Lyden, P. D., Knappertz, V. A., Ashwood, T., et al. (2004). Early impairment in consciousness predicts mortality after hemispheric ischemic stroke. Crit. Care Med. 32, 241-245. doi: 10.1097/01.CCM.0000104113.36384.81

Danielson, N. B., Guo, J. N., and Blumenfeld, H. (2011). The default mode network and altered consciousness in epilepsy. Behav. Neurol. 24, 55-65. doi: 10.3233/BEN-2011-0310

Di, X., Kannurpatti, S. S., Rypma, B., and Biswal, B. B. (2012). Calibrating BOLD fMRI activations with neurovascular and anatomical constraints. Cereb. Cortex 23, 255-263 doi: 10.1093/cercor/bhs001

Fernandez-Espejo, D., Soddu, A., Cruse, D., Palacios, E. M., Junque, C., Vanhaudenhuyse, A., et al. (2012). A role for the default mode network in the bases of disorders of consciousness. Ann. Neurol. 72, 335-343. doi: 10.1002/ana. 23635

Fischer, J., and Mathieson, C. (2001). The history of the Glasgow Coma Scale: implications for practice. Crit. Care Nurs. Q. 23, 52-58. doi: 10.1097/00002727200102000-00005

Frankel, M. R., Morgenstern, L. B., Kwiatkowski, T., Lu, M., Tilley, B. C., Broderick, J. P., et al. (2000). Predicting prognosis after stroke: a placebo group analysis from the National Institute of Neurological Disorders and stroke rt-PA stroke trial. Neurology 55, 952-959. doi: 10.1212/WNL.55.7.952

Gregoire, S. M., Charidimou, A., Gadapa, N., Dolan, E., Antoun, N., Peeters, A., et al. (2011). Acute ischaemic brain lesions in intracerebral haemorrhage: multicentre cross-sectional magnetic resonance imaging study. Brain 134, 2376-2386. doi: 10.1093/brain/awr172

Greicius, M. D., Flores, B. H., Menon, V., Glover, G. H., Solvason, H. B., Kenna, H., etal. (2007). Resting-state functional connectivity in major depression: abnormally increased contributions from subgenual cingulate cortex and thalamus. Biol. Psychiatry 62, 429-437. doi: 10.1016/j.biopsych.2006. 09.020

Greicius, M. D., Krasnow, B., Reiss, A. L., and Menon, V. (2003). Functional connectivity in the resting brain: a network analysis of the default mode hypothesis. Proc. Natl. Acad. Sci. U.S.A. 100, 253-258. doi: 10.1073/pnas.0135058100

Greicius, M. D., Srivastava, G., Reiss, A. L., and Menon, V. (2004). Defaultmode network activity distinguishes Alzheimer's disease from healthy aging: evidence from functional MRI. Proc. Natl. Acad. Sci. U.S.A. 101, 4637-4642. doi: 10.1073/pnas.0308627101

Hudetz, A. G. (2012). General anesthesia and human brain connectivity. Brain Connect. 2, 291-302. doi: 10.1089/brain.2012.0107

Kaisti, K. K., Metsahonkala, L., Teras, M., Oikonen, V., Aalto, S., Jaaskelainen, S., et al. (2002). Effects of surgical levels of propofol and sevoflurane anesthesia on cerebral blood flow in healthy subjects studied with positron emission tomography. Anesthesiology 96, 1358-1370. doi: 10.1097/00000542-20020600000015

Kannurpatti, S. S., Motes, M. A., Rypma, B., and Biswal, B. B. (2010). Neural and vascular variability and the fMRI-BOLD response in normal aging. Magn. Reson. Imaging 28, 466-476. doi: 10.1016/j.mri.2009.12.007

Lansberg, M. G., Lee, J., Christensen, S., Straka, M., De Silva, D. A., Mlynash, M., et al. (2011). RAPID automated patient selection for reperfusion therapy: a pooled analysis of the echoplanar imaging thrombolytic evaluation trial (EPITHET) and the diffusion and perfusion imaging evaluation for understanding stroke evolution (DEFUSE) study. Stroke 42, 1608-1614. doi: 10.1161/STROKEAHA.110.609008

Laureys, S., Lemaire, C., Maquet, P., Phillips, C., and Franck, G. (1999). Cerebral metabolism during vegetative state and after recovery to consciousness. J. Neurol. Neurosurg. Psychiatry 67, 121. doi: 10.1136/jnnp.67.1.121

Maquet, P., Faymonville, M. E., Degueldre, C., Delfiore, G., Franck, G., Luxen, A., et al. (1999). Functional neuroanatomy of hypnotic state. Biol. Psychiatry 45, 327-333. doi: 10.1016/S0006-3223(97)00546-5

Maquet, P., Peters, J., Aerts, J., Delfiore, G., Degueldre, C., Luxen, A., et al. (1996). Functional neuroanatomy of human rapid-eye-movement sleep and dreaming. Nature 383, 163-166. doi: 10.1038/383163a0

McNett, M. (2007). A review of the predictive ability of Glasgow Coma Scale scores in head-injured patients. J. Neurosci. Nurs. 39, 68-75. doi: 10.1097/01376517200704000-00002

Meindl, T., Teipel, S., Elmouden, R., Mueller, S., Koch, W., Dietrich, O., et al. (2010). Test-retest reproducibility of the default-mode network in healthy individuals. Hum. Brain Mapp. 31, 237-246. doi: 10.1002/hbm.20860
Ohta, T. (2005). Phenomenological aspects of consciousness - its disturbance in acute and chronic stages. Acta Neurochir. Suppl. 93, 191-193. doi: 10.1007/3-21127577-0_33

Park, C. H., Chang, W. H., Ohn, S. H., Kim, S. T., Bang, O. Y., PascualLeone, A., etal. (2011). Longitudinal changes of resting-state functional connectivity during motor recovery after stroke. Stroke 42, 1357-1362. doi: 10.1161/STROKEAHA.110.596155

Rainville, P., Hofbauer, R. K., Paus, T., Duncan, G. H., Bushnell, M. C., and Price, D. D. (1999). Cerebral mechanisms of hypnotic induction and suggestion. J. Cogn. Neurosci. 11, 110-125. doi: 10.1162/089892999563175

Ropper, A. H. (1986). Lateral displacement of the brain and level of consciousness in patients with an acute hemispheral mass. N. Engl. J. Med. 314, 953-958. doi: 10.1056/NEJM198604103141504

Sharp, D. J., Beckmann, C. F., Greenwood, R., Kinnunen, K. M., Bonnelle, V., De Boissezon, X., et al. (2011). Default mode network functional and structural connectivity after traumatic brain injury. Brain 134, 2233-2247. doi: 10.1093/brain/awr175

Soddu, A., Vanhaudenhuyse, A., Bahri, M. A., Bruno, M. A., Boly, M., Demertzi, A., et al. (2012). Identifying the default-mode component in spatial IC analyses of patients with disorders of consciousness. Hum. Brain Mapp. 33, 778-796. doi: 10.1002/hbm.21249

Soddu, A., Vanhaudenhuyse, A., Demertzi, A., Bruno, M. A., Tshibanda, L., Di, H., et al. (2011). Resting state activity in patients with disorders of consciousness. Funct. Neurol. 26, 37-43.

Steriade, M. (1996). Arousal: revisiting the reticular activating system. Science 272, 225-226. doi: 10.1126/science.272.5259.225

Teasdale, G., and Jennett, B. (1974). Assessment of coma and impaired consciousness. A practical scale. Lancet 2, 81-84. doi: 10.1016/S0140-6736(74)91639-0

Uddin, L. Q., Supekar, K., Amin, H., Rykhlevskaia, E., Nguyen, D. A., Greicius, M. D., et al. (2010). Dissociable connectivity within human angular gyrus and intraparietal sulcus: evidence from functional and structural connectivity. Cereb. Cortex 20, 2636-2646. doi: 10.1093/cercor/bhq011

Vanhaudenhuyse, A., Demertzi, A., Schabus, M., Noirhomme, Q., Bredart, S., Boly, M., etal. (2011). Two distinct neuronal networks mediate the awareness of environment and of self. J. Cogn. Neurosci. 23, 570-578. doi: 10.1162/jocn.2010.21488

Vanhaudenhuyse, A., Noirhomme, Q., Tshibanda, L. J., Bruno, M. A., Boveroux, P., Schnakers, C., et al. (2010). Default network connectivity reflects the level of consciousness in non-communicative brain-damaged patients. Brain 133, 161171. doi: 10.1093/brain/awp313

Wang, L., Dai, W., Su, Y., Wang, G., Tan, Y., Jin, Z., et al. (2012). Amplitude of low-frequency oscillations in first-episode, treatment-naive patients with major depressive disorder: a resting-state functional MRI study. PLoS ONE 7:e48658. doi: 10.1371/journal.pone.0048658

Wang, X., Jiao, Y., Tang, T., Wang, H., and Lu, Z. (2013). Altered regional homogeneity patterns in adults with attention-deficit hyperactivity disorder. Eur. J. Radiol. 82, 1552-1557. doi: 10.1016/j.ejrad.2013.04.009

Weir, C. J., Bradford, A. P., and Lees, K. R. (2003). The prognostic value of the components of the Glasgow Coma Scale following acute stroke. QJM 96, 67-74. doi: 10.1093/qjmed/hcg008

Wu, T., Long, X., Zang, Y., Wang, L., Hallett, M., Li, K., et al. (2009). Regional homogeneity changes in patients with Parkinson's disease. Hum. Brain Mapp. 30, 1502-1510. doi: 10.1002/hbm.20622

Yuan, R., Di, X., Kim, E. H., Barik, S., Rypma, B., and Biswal, B. B. (2013). Regional homogeneity of resting-state fMRI contributes to both neurovascular and task activation variations. Magn. Reson. Imaging 31, 1492-1500. doi: 10.1016/j.mri.2013.07.005

Zang, Y. F., He, Y., Zhu, C. Z., Cao, Q. J., Sui, M. Q., Liang, M., et al. (2007). Altered baseline brain activity in children with ADHD revealed by resting-state functional MRI. Brain Dev. 29, 83-91. doi: 10.1016/j.braindev.2006.07.002

Zhang, Z., Liu, Y., Jiang, T., Zhou, B., An, N., Dai, H., et al. (2012). Altered spontaneous activity in Alzheimer's disease and mild cognitive impairment revealed by regional homogeneity. Neuroimage 59, 1429-1440. doi: 10.1016/j.neuroimage.2011.08.049

Zhou, Y., Liang, M., Jiang, T., Tian, L., Liu, Y., Liu, Z., et al. (2007). Functional dysconnectivity of the dorsolateral prefrontal cortex in first-episode schizophrenia using resting-state fMRI. Neurosci. Lett. 417, 297-302. doi: 10.1016/j.neulet.2007.02.08 
Conflict of Interest Statement: The authors declare that the research was conducted in the absence of any commercial or financial relationships that could be construed as a potential conflict of interest.

Received: 27 May 2013; accepted: 04 December 2013; published online: 02 January 2014.

Citation: Tsai Y-H, Yuan R, Huang Y-C, Yeh M-Y, Lin C-P and Biswal BB (2014)

Disruption of brain connectivity in acute stroke patients with early impairment in consciousness. Front. Psychol. 4:956. doi: 10.3389/fpsyg.2013.00956
This article was submitted to Consciousness Research, a section of the journal Frontiers in Psychology.

Copyright (c) 2014 Tsai, Yuan, Huang, Yeh, Lin and Biswal. This is an openaccess article distributed under the terms of the Creative Commons Attribution License (CC BY). The use, distribution or reproduction in other forums is permitted, provided the original author(s) or licensor are credited and that the original publication in this journal is cited, in accordance with accepted academic practice. No use, distribution or reproduction is permitted which does not comply with these terms. 